\title{
A PILOT STUDY TOWARDS RANKING OCCUPATIONAL HEALTH RISK FACTORS EMANATING FROM ENGINEERED NANOPARTICLES: REVIEW OF A DECADE OF LITERATURE
}

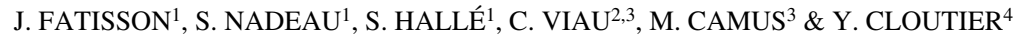 \\ ${ }^{1}$ Mechanical Engineering, École de Technologie Supérieure, Montreal, Quebec, Canada. \\ ${ }^{2}$ Institut de Recherche en Santé Publique, Université of Montreal, Montreal, Quebec, Canada. \\ ${ }^{3}$ Occupational and Environmental Health Department, Université of Montreal, Montreal, Quebec, Canada. \\ ${ }^{4}$ Institut de Recherche Robert Sauvé en Santé et Sécurité du Travail, Montreal, Québec, Canada.
}

\begin{abstract}
As beneficial applications of nanotechnologies in industry and medicine continue to emerge, so do new problems associated with engineered nanoparticle (ENP) production, which so far is going ahead without prior evaluation of its impact on human health and environment. Worker exposure continues to increase while no global consensus on ENP regulation has been reached. Protection of workers requires an approach to risk management properly adapted to the ENP context. Although ENP properties have been studied in depth over the past 10 years, much uncertainty continues to loom over the definition of the key parameters. The aim of this review of the literature was to construct a detailed list of known risks associated with ENPs from an occupational health and safety perspective. A hierarchised network of risks was thus revealed, illustrating the complexity of the system in terms of interdependence of elements of risk.

Keywords: Engineered nanoparticle, hierarchised network, risk assessment, risk categorisation, risk management.
\end{abstract}

\section{INTRODUCTION}

'Nanotechnology consumer products are in your mouth and on your face'. This affirmation dates back at least to April 24, 2008, when it was surmised from the list maintained by the Project on emerging nanotechnologies [1] that new products were being introduced into the market at a rate of three or four per week, a trend that has since accelerated. The global market for nanomaterials is expected to exceed 2400 billion $\$$ US by 2015 [2]. Each day, science demonstrates the usefulness of nanotechnologies, and the production of nanomaterials is now viewed as a major contributor to the next industrial and medical revolution. However, in spite of their beneficial properties, these new materials also raise suspicions, in particular regarding new problems relating to human health, while many industries are plunging headlong into production without prior evaluation of the impact of their materials or products on health or the environment.

Nanotechnologies are involved in almost all major sectors of production: construction, automobiles, aeronautical industry, textiles, energy, electronics, medical industries, agri-food and so on. NanoQuébec in 2008 enumerated an estimated 573 nanotechnological industries in the USA and 51 in Canada. In 2011, Nanowerk [3] counted 1168 commercial organisations active in nanotechnologies in USA and 86 in Canada. Industry Canada counts 21 Quebec manufacturers of nanotechnology products. As the nanotechnology market continues to grow, so does the number of workers continually exposed to nanoparticles. This number remains difficult to determine, although it appears to be very large and could reach two million within 10 years $[4,5]$. Based on global surveys, as many as two million new jobs 
related to nanotechnologies could be created by 2015 [6]. This phenomenon appears to involve small-to-medium-sized businesses as well as multi-nationals. Meanwhile, there is still no regulatory system in place, no doubt due to the absence of a clear definition of nanomaterials, although numerous efforts addressing this situation are noted [7] and a broad definition has been proposed recently in Europe [8]. At the same time, organisations, such as the Environmental Protection Agency [9], the World Health Organization with resolution II/4 [10] or the European Commission with the REACH framework [11], have already started to propose regulations on nanobased products. However, no global consensus has been reached yet, while the number of businesses producing nanomaterials continues to grow and the notion of worker protection in this field remains nebulous. Some observers have reached the conclusion that the difficulties encountered in attempting to define the potential impact of ultra-fine particles on human well being are major [12, 13].

For the past several years, the number of studies of the physical, chemical and biological properties of nanoparticles has grown in proportion with the associated economic activity. Based on PubMed, the number of publications matching keywords such as ultrafine particles or nanoparticles plus toxicity or effects on health was 5 in 2001, 246 in 2006 and 1516 in 2011. However, much uncertainty remains and limits the understanding of their mechanisms of toxic interaction with the human body as well as conditions of exposure [14, 15]. The understanding of nanotoxicity [16] is evolving fast as well as the science of the characterisation of these risks, while nanotechnologies follow and evolve just as quickly.

The uncertainty surrounding nanomaterials is due also to a lack of standardisation of characterisation procedures and toxicological testing methods [17-21]. Some research groups [22], the Organisation for Economic Co-operation and Development (OECD) and other standards organisations [23-26] are developing procedures and other guidelines for the use and the characterisation of nanomaterials in order to help fill this void. Even if, synthetic nanomaterials are legally defined as substances by REACH regulation (EC regulation no 1907/2006) [11] but REACH framework might not be adapted for nanomaterials. Therefore, evaluation of health and safety or environmental risks more specific to nanomaterials remains necessary (COM/2008/0366 final) [27]. In the absence of global consensus, no recent protocol regarding regulation has been implemented [22], in spite of efforts by the Occupational Safety and Health Organization. The comparison of studies, therefore, remains very difficult and the task seems insurmountable given the multitude of nanoparticle types, now compounded by the possibility of mixing different types for specific applications. While we wait for toxicological results and the development of characterisation techniques, Québec applies the precautionary principle in limiting harmful effects to a certain extent while not closing the door to benefiting from the development of new nanomaterials [28].

However, precautionary principle, as well as classic risk management methods, seems unsuitable in front of the challenges brought by nanotechnology [29]. Given the speed at which nanotechnologies are developing, new risk assessment and management methods are required. As emphasised by Aitken et al. [21], risk evaluation methods are inadequate and require better use of the data available as well as the acquisition of new data. New methods of risk management must be implemented in order to protect individuals who enter into contact with engineered nanoparticles (ENP) one way or another. The first step in the development of risk management tools involves cataloguing and classifying all risk factors associated with nanoparticles. Kara Morgan [30] has, thus, developed a basic framework for guiding the analysis and management of risks to human health and the environment due to nanoparticles. This excellent work was completed 8 years ago and should be pursued. Meanwhile, ENP 
toxicity studies have grown tenfold (401 publications between 2001 and 2005 and 4542 between 2006 and 2011). In order to preserve the essential human perspective on the complexity of the problem, we have devoted our literature review to ENP. This type of nanomaterial is the single most common type and involves the largest number of workers and exposures to the highest concentrations. Once the implications for the health of workers are understood and suitable protective measures are implemented, similar monitoring could be implemented for consumers in general. In fact, considering the diversity of ENP and their applications, a case-by-case approach would be a very time-consuming process. Nadeau et al. [31] recently argued that the development of a flexible, easy to use, dynamic and integrated tool incorporating reliable data on the risks associated with nanoparticles was urgent. Several others have also recommended this strategy [32, 33].

For the analysis of risks associated with chemicals, the first step is hazard identification [34]. Transposing this notion to nanoparticles, which may be considered as chemicals [11], amounts to listing all hazard and exposure factors and effects. The aim of the present review was, therefore, to categorise the risk factors currently associated with ENP in the workplace in order to facilitate the development of a decision-aid tool intended for producers of synthetic nanoparticles and for specialists in preventive measures. Eight major groups of risks were thus identified in the literature and represented in a hierarchised risk network in order to portray the complexity of the system. This work thus illustrates clearly the interdependence between the elements of risk as well as needs regarding the evaluation of certain risks, for example, the need to integrate exposure data. The results obtained in this pilot study will serve as a basis for further developments and discussions.

\section{METHODOLOGY}

\subsection{Review of the literature}

This literature review was conducted according to a previously described widely adopted systematic protocol [35]. The research questions were as follows: Over the past decade, what risk factors associated with nanoparticles have been identified and what associations exist between them? Using PubMed database and the SciFinder scholarly research tool, we conducted the systematic literature review for the years 2001-2011 using the following keywords: nanoparticle, nanomaterial, risk, uncertainty, risk management, risk assessment, health effect, toxicity, in various combinations. To focus the process, review articles and commission reports were first selected, followed by research articles. References cited in these papers were used to identify additional studies published during the 2001-2011 period. In order to refine the results, a descending method was used initially, taking advantage of recent secondary bibliographic sources. The references in these sources allowed us to reach the primary bibliographic sources and improve the list of keywords used. In view of the large number of studies, we then reverted to an ascending method allowing us to refine the search and keep this literature database continually updated. Through rigorous examination of each article, we listed the risk factors identified by the authors of the studies. Articles not published during the selected period were excluded, as were those judged not relevant to the research question, thus reducing interpretation biases and highly deficient protocols.

Following this initial step, we continued the literature review using an iterative process conducted in three steps (Fig. 1) and which followed an approach based on creating a fault tree [36]. We first classified all occupational health-related risk elements (Step 2), on the basis 


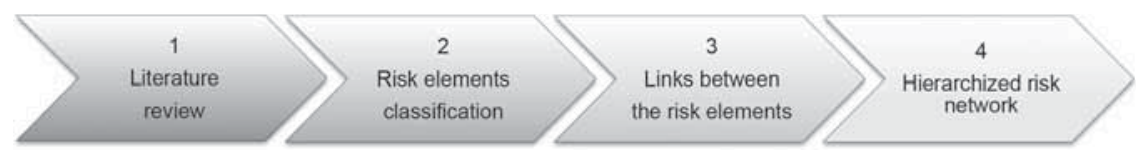

Figure 1: Four-step risk assessment method used to develop a decision-aid tool prototype.

of whether the factor was associated with an intrinsic parameter (source), with a transfer to target (exposure) factor or with a harmful effect (target) [30, 37, 38]. In a third step, using specific keywords corresponding to each variable listed, we identified all observed links between all individual risk factors (Step 3). All observations and data from this review were further compiled and associations between risk factors were arranged in a hierarchised network (Step 4).

We could produce an additional grouping into eight different categories at the end of step 4: intrinsic properties of ENP, interfacial properties, mobility-related properties, taskrelated exposure factors, ambient exposure factors, physiological effects on the body, specific effects on cells and other harmful effects. Each of these categories is described in detail in the following section. The purpose of this grouping was also to simplify the graphic representation of the network of associations existing between the various factors and effects. The expression 'elements of risk' refers to hazards, factors of transfer to the target and effects.

\subsection{Influence diagram}

Two types of approach were taken to estimating influences among risk factors and effects: $a$ priori and a posteriori. Since epidemiological studies of nanoparticles are so scant, the preferred approach would appear to be a priori, which estimates the possible associations between causes and consequences before undesirable events occur.

The purpose of an influence diagram, commonly called a risk tree, is to provide a graphic representation of cause-consequence associations existing between various elements of risk, as well as the harm associated with the risks. There are several techniques based on causes, consequences or events for connecting causes to consequences. A fault tree is a logical diagram that shows the relationships leading to an undesirable event. This approach is easy to use, favours systematic search for causes and their associations and does not require chronological relations between risk factors and effects [39].

As will be described in greater detail below, fault trees as defined [36] need to be converted to a hierarchised network of risks [40]. Other more complex modelling systems also will be required in order to develop an adequate and adaptable decision-aid tool.

Arrows between elements of risk (Fig. 2) indicate cause-consequence associations; the arrow heads indicate which element is influenced or that influence is mutual. Arrow thickness represents the importance of the associations, based on an initial estimation of the number of studies reporting the influence of a given factor on another or on an effect. This enumeration was not exhaustive, in any case, since several reviews of the literature have reached the conclusion that this would be a colossal task. However, the bare number of studies discussing a factor is not necessarily a reliable indicator of its importance. Certain instrumentation, testing techniques and analytical possibilities were unknown 10 years ago. It was easier to measure the size of nanoparticles using microscopy than to perform real-time analysis of particle agglomeration rates in matrices identical or nearly identical to those used in cytotoxicity studies. 


\section{DEVELOPMENT}

\subsection{Elements of risk}

In order to simplify the exercise, each element was assimilated into a more general factor, based on its definition in the literature (see Table 1). For example, optical, thermal or mechanical properties were grouped into a single factor called physical properties. All identified elements of risk were first grouped into three principal categories (level-1 classification) based on whether they were part of a danger factor, a parameter stemming from exposure (transfer to the target) or an effect. To date, 14 factors representing occupational health and safety (OHS) hazards have been listed as inherent in nanoparticles (level-3 classification).

\subsubsection{ENP hazards}

Grass et al. [41] demonstrated that risks associated specifically with nanoparticles stem ultimately from three sources: large interfacial area, the possibility of quantum effects and non-conventional mobility factors (agglomeration, toxicokinetics, translocation, accumulation). However, their studies were essentially conducted in vitro and did not take exposure factors into consideration. Furthermore, certain effects cannot be studied in vitro. A second classification of hazards was nevertheless completed. Certain parameters were thus considered intrinsically associated with nanoparticles, others with their surface or interfacial properties or with their mobility properties. In addition, as noted by Oberdorster et al. [42], the mechanisms of nanoparticle toxicity are now being studied in terms of mobility properties and surface interactions. Table 1 lists the 14 hazards and sub-hazards, as well as references that describe these parameters as important for the evaluation of risks associated with nanoparticles.

According to a review by Grieger et al. [19], general or intrinsic characteristics of nanomaterials are the second most significant source of uncertainty in risk evaluation. These include the most studied and reported characteristic, namely nanoparticle size. Several means of defining nanoparticle size exist: measurement of primary dimensions (often by transmission electron microscopy or TEM), average size or even size distribution, as described in several reports mentioned in Table 1. The OECD has included size distribution in the gaseous phase and in liquid medium in the list of major physicochemical properties to be characterised for ENP risk evaluation purposes [25]. The Scientific Committee on Emerging and NewlyIdentified Health Risks (SCENIHR) has also identified size as a parameter to be characterised [43]. Exposure to airborne particles is defined in terms of inhalation of various size categories (also called fractions) [44]. However, size is not necessarily the principal characteristic determining the mechanisms leading to particle toxicity. It is, therefore, necessary to create a tool for defining and ranking the risks inherent in each ENP. In fact, ENP size characterisation should be associated with nanoparticle shape. Whether cited as shape, aspect ratio or morphology, this factor may be determinant in interactions among particles or with other substrates, biological or otherwise. Furthermore, according to the definition proposed by the Royal Society, 'nanotechnology is the production and application of structures, devices and systems by controlling shape and size at the nanometre scale' [45]. This definition places ENP shape in a preponderant position. Aitken et al. [21] and Paik et al. [46] also noted the relevance of shape to proper evaluation of nanoparticle properties.

From a combination of physical dimensions (size and shape), it is possible to determine the surface area. This characteristic may be considered as a risk factor, since several research 


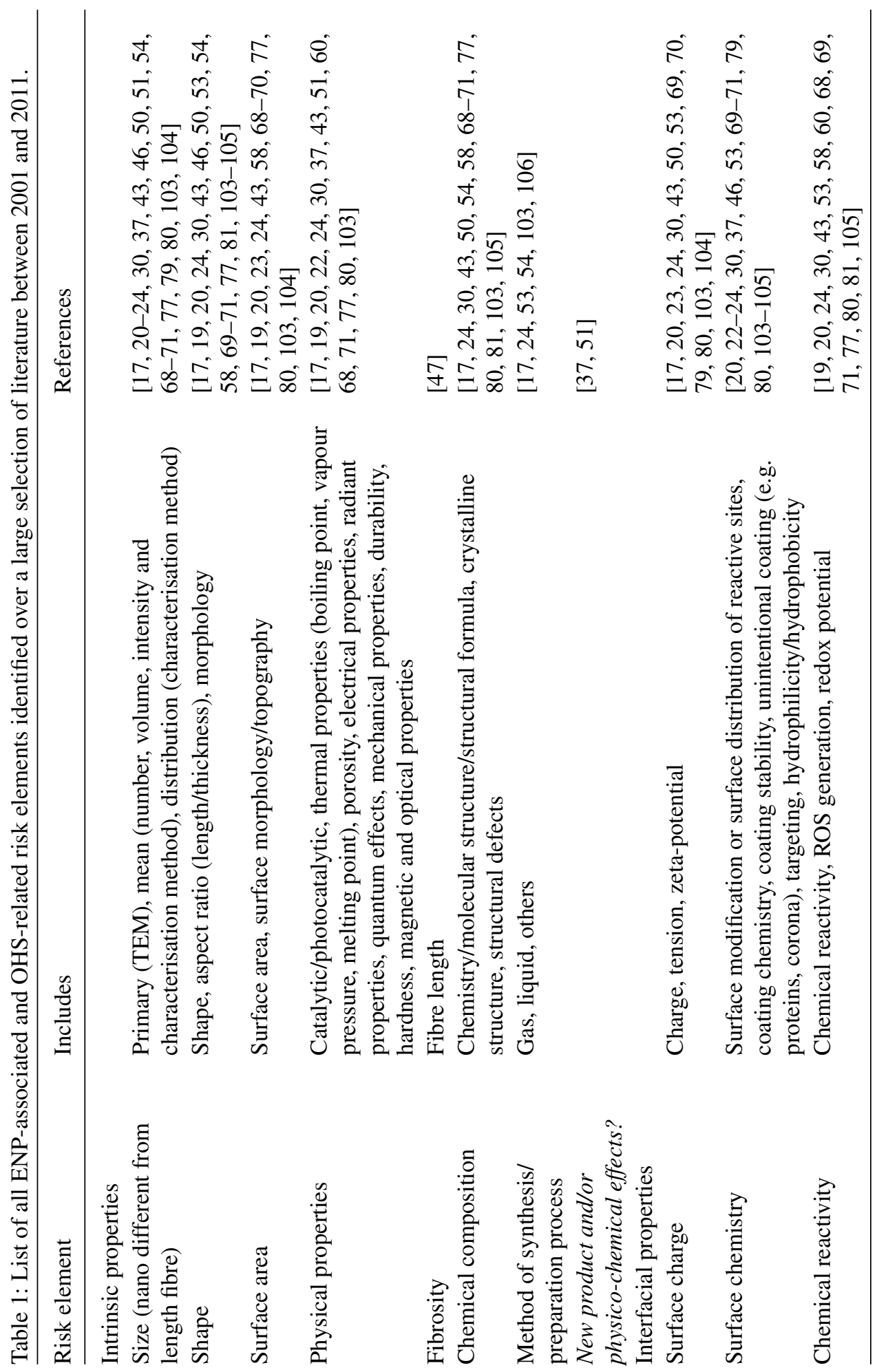




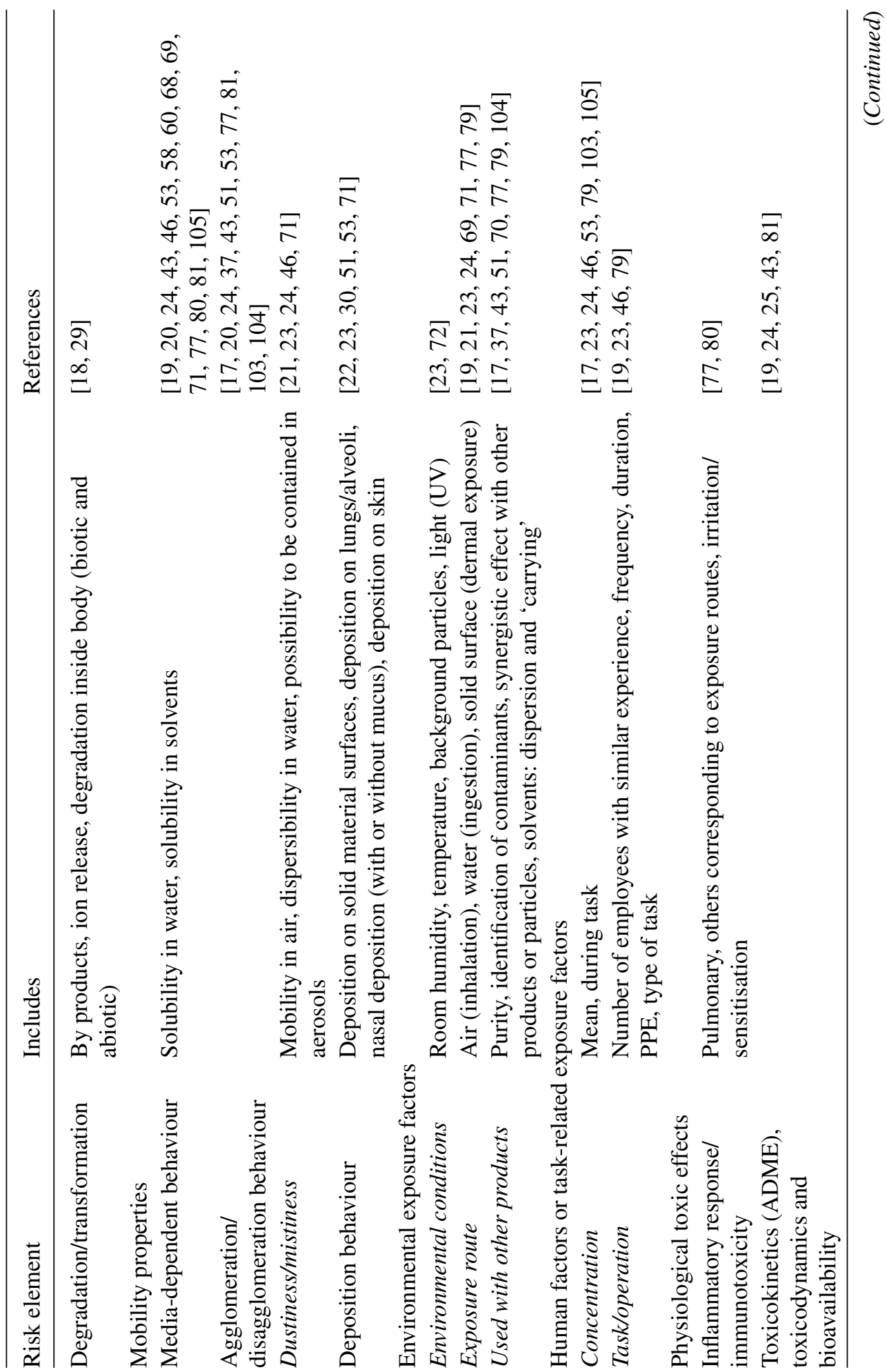




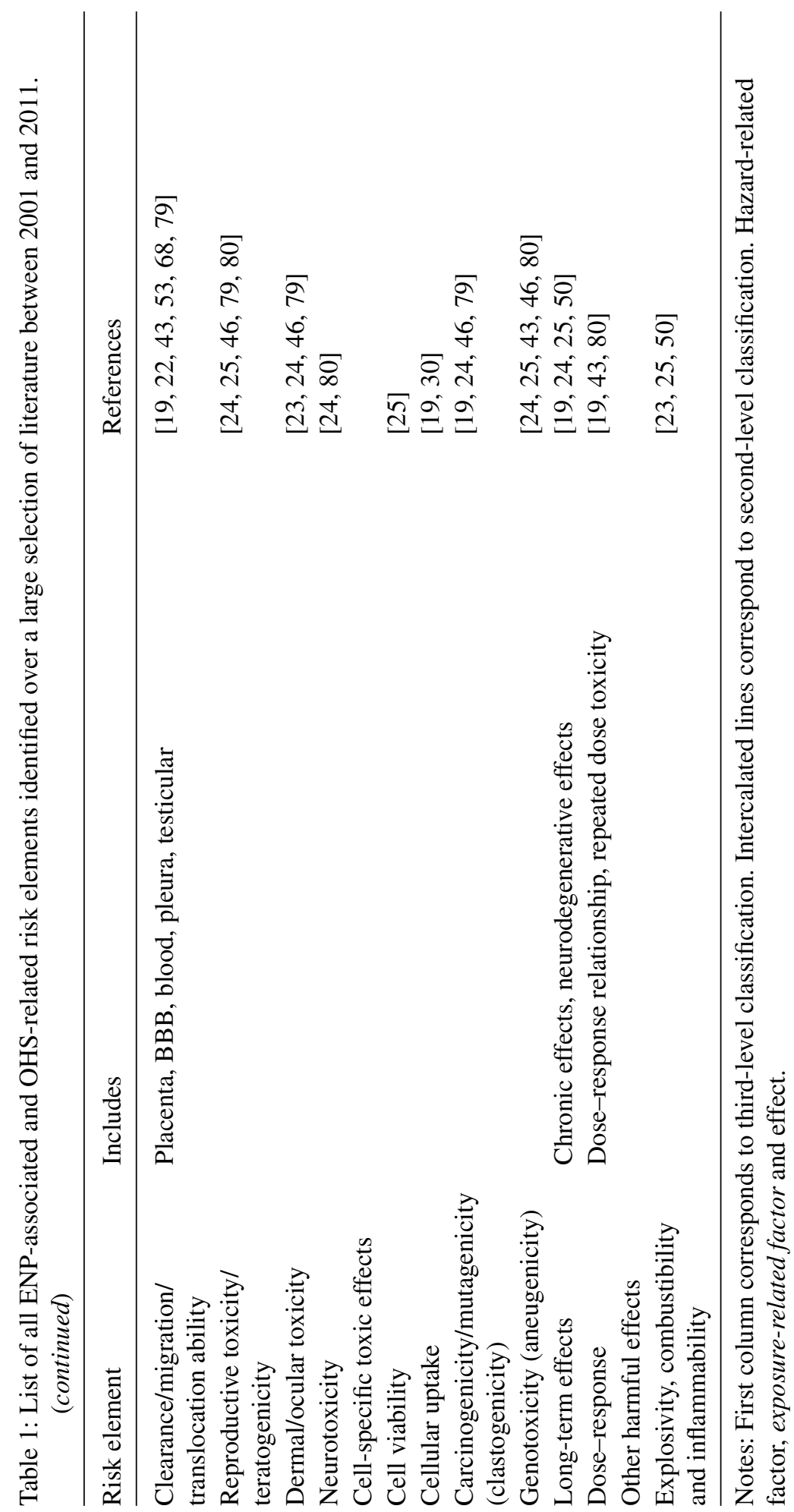


groups and organisations have identified it as a standard of characterisation, as well as a term for defining exposure conditions [21, 25, 43]. In addition, based on a Delphi-type survey, experts chosen by Berube et al. [47] ranked surface area as the fourth out of 14 problematic characteristics peculiar to nanoparticles. In view of the broad scope of proposed European recommendations for the definition of nanomaterials, dimensions outside of the nanometre scale could also be included [8]. Numerous reports describe dangers associated with particle fibrous character such as asbestos [48]. This characteristic is currently applicable to carbon nanotubes [49], although other materials could also be processed into fibres or produced as such.

Size, shape and surface area have direct influences on the various physical properties of nanoparticles. Among these properties are thermal, mechanical, catalytic, optical, radiant and magnetic properties, as listed in Table 1. Different research groups have identified these properties as potential elements of risk. For example, ENP electrical or thermal conductivity should be considered when choosing or designing the area in which these materials are produced, and proper monitoring, insulation and isolation of workers should be provided. Aitken et al. [21] have used physical properties (electrical and magnetic among others) to categorise various types of nanoparticles in a review of workplace hygiene. Aside from physical factors, ENP chemical characteristics, as well as the structural defects, could also influence other properties, as well as affect particle toxicity. Furthermore, these factors have been identified by nanoparticle-risk-monitoring organisations as essential for ENP evaluation [25, 43, 50]. Other authors $[37,51]$ have also examined the possibility that new nanoproducts have yet unknown physical or chemical properties and therefore represent unknown risks to human health. A recent study has shown the feasibility of using a multi-criteria method to evaluate risks associated with a carbon nanotube synthesis pathway [52]. Cited in other reviews of the literature [21, 53, 54], this report suggests that ENP synthesis methods should be considered as an intrinsic risk factor in the sense that they have physical, chemical and biological consequences.

High-risk properties of ENP products could be examined from the perspective of their potential for interactions with other objects, which may be living or not [41]. Among the more notable interfacial properties cited in the literature are interfacial tension and surface charge or zeta potential. According to the well-known theory of Derjaguin-Landau-VerweyOverbeek, the principal forces involved during interaction between two objects are electrostatic. It is, therefore, plausible that interactions among ENP products or with living cells will depend on net charge, even if it is known that cells usually bear a negative charge. Another category of well-studied and often cited interfacial properties is concerned with surface chemistry, the surface distribution of chemical sites and the coating of ENP products, intentional or otherwise (Table 1). During contact between ENP products and a biological material, the nanoparticles may become coated with molecules from the extracellular milieu, such as proteins or other ionic substances. Such unintentional coating may change the ENP surface chemistry [55-58] and consequently modify attractive or repulsive forces between ENP bodies or between these and cells. A wide variety of other mechanisms must, therefore, be taken into consideration when evaluating ENP toxicity.

Other properties associated with ENP chemical reactivity but not identified in other categories may also be important, such as redox potential. The capacity of metallic ENP products to generate reactive oxygen species (ROS) based on redox potential $[59,60]$ often appears plausible as a mechanism explaining their cellular toxicity or inflammatory reactions. Another chemical phenomenon cited by Morgan [30] and Grieger et al. [19] stems directly from 
chemical reactivity and should be in its own category. This concerns the potentially toxic breakdown products from reactions between ENP products and their targets. Using the example of quantum dots, it has been shown that dissolving the core of these particles liberates cadmium ions, which are recognised as highly toxic [61], while the particles themselves are not necessarily as toxic. Similar properties have been shown in the case of other ENP products $[62,63]$.

The third principal category of intrinsic properties of ENP products is related to their mobility and associated characteristics. Physical, chemical and interfacial properties stem from ENP behaviour in different media. For example, water-dispersible ENP products will not have the same effects as more hydrophobic products on biological organisms. Depending on their solubility, their mobility calculated on the basis of diffusion in a given medium [41] can have implications for possible interactions with cells or extracellular macromolecules. They also may or may not aggregate or form stable colloids. Agglomeration is often cited as an essential standard of characterisation (Table 1). In fact, the nominal size of ENPs as measured by TEM may provide little information about their behaviour in the aqueous phase [64]. For example, the mechanisms of entry of individual particles or aggregates into cells can differ, depending on plasma membrane pores [65].

Another factor, considered more of an exposure factor, may also be included in this category, given that it too depends on ENP interfacial properties. The propensity of ENPs to form dust or aerosols becomes particularly significant when adapting ENP manufacturing ventilation systems. In addition, the tendency of some ENP products to accumulate on surfaces is a strong indication that they are suspended in air or in moisture suspended in air or likely to become so. This is another characteristic that needs to be evaluated since contact between ENPs in the air, on surfaces (e.g. work plans) and the human body becomes possible. The literature is replete with recommendations to confine and contain synthetic nanoparticles by process design $[66,67]$, during operations, during maintenance and in situations of accidental spills or potential aerosol formation [68-70]. Understanding ENP behaviour should make it possible to determine the most probable entry routes and thereby facilitate rational design, implementation and improvement of monitoring and control measures intended to reduce exposure via these pathways $[21,41,46,71]$.

\subsubsection{Exposure factors}

Having described the principal ENP characteristics and properties that make them potentially hazardous, before discussing harmful effects we must examine the transfer factors that allow particles to enter into contact with workers. We have grouped these into two principal categories (level-2 classification), namely environmental factors and task-related factors (production, clean-up, etc.).

The first factors to consider are those associated with the environment in which ENP products are produced or otherwise occur. Murashov et al. [72] have cited certain conditions to consider with respect to measures addressing not only exposure, namely ambient atmospheric moisture (potential for the production of ENP aerosols), temperature (which influences particle mobility), light (it is known that $\mathrm{TiO}_{2}$ particles can be toxic after activated by UVVisible light $[73,74])$, but also the presence of other background particles. Unintentionally produced nanoparticles occur in the air we breathe, such as diesel particles and particles produced during machining [75], and can potentially affect the behaviour of nanoaerosols. Several studies of workplace exposure [76] show that it is often difficult to distinguish ENP products from background particles. Background particles can agglomerate with ENPs and 
therefore affect ENP mobility, which we have already included in our list of intrinsic elements of risk.

To these factors we must add the path of exposure by which ENPs will enter into contact with the human body $[21,77]$. The toxic mechanism (organs most affected) will vary depending on whether the particles are inhaled (are present in the ambient air), ingested or in contact with the skin or eyes. Inhaled particles will enter the lungs and reach the blood or may reach the brain via the olfactory nerve [78], whereas ingested particles will first be exposed to the gastro-intestinal tract content before potential absorption and distribution to many organs. Passage through the skin also allows them to gain access to the bloodstream.

During manufacture, ENPs can also become mixed (intentionally or otherwise) with other products. The SCENIHR, therefore, recommends measuring ENP purity [43]. The presence of contaminants, which may also be toxic, could influence ENP toxicity. It should also be determined to what extent evaporating solvents can carry ENPs and thus introduce them into the ambient air.

In addition to environmental factors per se, parameters associated directly with ENP production or handling tasks should also be taken into consideration. As is the case for any product, the human body likely has mechanisms for dealing with particles up to some point and the definition of ENP toxicity will therefore include some concentration [6, 23, 43, 50]. ENP concentrations in ambient air may be a direct consequence of a task and the conditions under which the task is carried out. Worker exposure will depend on the duration and frequency of the task, the number of workers present and the type of task (manufacturing, packaging, stock management, clean-up, etc.). These factors have been discussed in scientific reports based on field studies [79] or have been examined during the development of monitoring tools for ensuring worker safety [46] or in previous reviews of the literature [19].

\subsubsection{Effects}

When evaluating ENP toxicity, several parameters must be examined in order to determine which mechanism to consider for the purpose of defining the particles as toxic or not. Depending on the intrinsic properties listed above and on transfer factors, the effect to consider and sometimes the evaluation method as well may differ. The effects category thus may be divided into cell-based, whole body or indirect appreciations of the harmfulness of ENPs to human beings. Measurements of cell viability following contact with ENPs are the subject of multitude of studies and reports on the acute toxicity of numerous ENPs under different conditions intended to shed light on the role of particle intrinsic properties. Much of the research has been devoted to examining ENP entry into cells in quantitative terms in order to determine by what mechanisms this occurs and what the intracellular effects are. The mechanisms by which damage is inflicted upon the cells have also received much attention. While other parameters remain shrouded in uncertainty, cellular uptake is one of the most studied ENP characteristics, according to a recent inquiry by Grieger et al. [19].

Other institutional reports $[25,43,80]$ mention genotoxicity tests as necessary for proper toxicological evaluation of ENPs. Other authors $[46,81]$ have also mentioned this as well as other types of toxicity (carcinogenicity or mutagenicity) as possible non-negligible effects of ENPs. In general, organisations and institutions mandated to protect the population as a whole and workers in particular also recommend characterising cellular responses to acute, repeated and even chronic exposure. However, while these organisations attempt to establish testing standards, no consensus has yet emerged on the metric to use (particle diameter, surface area, mass, etc.) [20,77], and this remains a major methodological challenge for risk evaluation [82]. 
More holistic approaches to the evaluation of ENP toxicity may also be useful. Although it is important to understand the mechanisms by which the particles enter cells, this information alone is insufficient. Many studies report inflammatory effects of ENPs, and as mentioned by Aitken et al. [21], one of the challenges in toxicology is the quantification of these effects. The inflammatory response remains a key element of the response to contact with materials in general and helps to determine genotoxic potential [43]. Due to its non-specific nature, the inflammatory response is difficult to determine and quantify.

It appears just as relevant to consider the overall impact of ENPs on the human body. Toxicokinetic and toxicodynamic studies of ENPS have been conducted using the widely practised absorption, distribution, metabolism and excretion (ADME) approach. In order to protect workers in contact with ENPs, it appears necessary to understand how nanoparticles enter, circulate, are metabolised and excreted by the body as a whole as well as by specific organs. The OECD and ISO recommend this approach [24, 25]. Several research groups emphasise the importance of examining organ-to-organ migration or system-to-system migration via blood vessels [83] or even via nerves [78] as possibilities for ENP effects in the human body. Effects on the nervous system, vision, reproduction and skin function should thus be considered. As observed by Oberdorster et al. [42] the approach to studying ENP toxicology is evolving and diversifying. The increasing number of uncertainties is making the evaluation of ENP-associated risk ever more challenging, a challenge that will only be met by considering as many risk factors as possible.

Aside from effects due to direct interaction between ENPs and the human body, there are other potential effects that represent major threats to worker safety. Various protection and standardisation organisations $[24,25,50]$ recommend tests for verifying ENP explosiveness, combustibility and flammability.

\subsection{Associations between factors}

The aim of this review was not to produce an exhaustive list of the publications that reveal associations between each factor (intrinsic or exposure) or with the listed effects. Using the categories listed in Table 1 as keywords for searches conducted according to the approach developed in Section 2 (search tools, period consulted), some insight into associations and their relevance was gained.

\subsubsection{Between each factor (level-3 classification)}

The first step of phase 3 (Fig. 1) consisted of identifying the existing associations between intrinsic properties that have been the subject of studies or reports. In the present review, we are concerned with elements of risk considered individually and only the relationships judged relevant by the authors of these studies or reports. Quantifying the impact of these relationships would require in-depth meta-analysis of all of these relationships.

It is now recognised that the size of ENPs confers them properties that are quite different from those of their micron-size equivalents. This is reflected in the number of studies devoted to the effect of size on the various physical and chemical properties of ENPs described in the previous section (Table 1). As mentioned by Auffan et al. [60], 30-40\% of the atoms of ENP spheres, $10 \mathrm{~nm}$ in diameter, are at the particle surface compared with only $20 \%$ for $30 \mathrm{~nm}$ particles. In another study, these researchers demonstrated the impact of size on nanoparticle crystallography using a thermodynamic approach [84]. Borm et al. [54, 85] produced a non-exhaustive list of the influence of various ENP properties on other general parameters. 
Initial contact between ENPs and the human body involves adsorption of various proteins and enzymes [56, 57, 65] on the nanoparticle surface. Studying this aspect, Vertegel et al. [86] demonstrated that the size of silica nanoparticles can affect the structure and activity of lysozyme, a bacteriolytic enzyme found in saliva and tears. Protein conformation can be influenced by the nanoparticle form [55]. Another factor that also influences adsorption of proteins is the presence of coatings on the ENP surface, which could have a direct impact on cell-mediated responses [87-89].

The influence of ENP size on cytotoxicity or general toxic effects (inflammatory reaction) has been the subject of numerous studies. Other parameters such as crystalline structure may also have an impact on the mode of cell death, as demonstrated by Braydich-Stolle et al. [90]. Meanwhile, other teams have focused more on parameters such as shape and surface chemistry $[91,92]$ and their impact on particle internalisation by cells or on intracellular traffic in order to gain better understanding of the ENP intracellular toxic process. Although the surface coating question has led to contradictory answers, it remains an aspect that should be studied in greater depth.

Very little attention has been given to the study of long-term effects stemming from ENP breakdown. Some researchers have examined the effects of free radicals on ENP genotoxicity [93], whereas others have discussed the impact of ion shedding on cytotoxicity [60, 94, 95].

In relatively recent research, several groups have noted that ENPs display translocation capability $[54,78,83,94,96]$. This is an alarming observation and has received much attention. Kreyling et al. [96] have demonstrated that the translocation of iridium particles from epithelial cells towards extra-pulmonary organs, though minimal, depends on particle size. The role of intrinsic and exposure factors should be studied in depth with other types of ENP, especially in the case of translocation through the lung lining into the bloodstream [54]. Simko and Mattsson [94] note that this property could have neurotoxic implications. The SCENIHR [43] mentions this aspect as an integral part of the evaluation of risks posed by nanotechnologies.

Several relationships between elements of risk (intrinsic factors, exposure factors, effects) have yet to be studied. This may be due to a perceived lack of relevance of certain factors, low priority at research funding organisations, instrumentation limitations, the succinct characterisation of ENPs tested in numerous studies or limitations on comparisons between studies of similar type due to poor standardisation of techniques. For example, we have not found evidence of direct relationships between ENP size and shape. However, as already noted, size and shape are directly related to surface area, which is in turn a factor in chemical reactivity and hence the potential toxicity of ENPs.

Important relationships need not be direct. In order to establish the more obvious connexions, risk-ranking tools are necessary. After producing an initial graphical representation (not shown) of all of the associations listed, we then grouped all of these elements into subcategories for clarity.

\subsubsection{Between the eight level-2 categories}

Once all of the associations mentioned in the literature between the various intrinsic and exposure factors and effects were represented in matrix form (Table S1), the various level-3 categories were grouped by level-2 category. The relationships between the different factors were represented using arrows, producing the graphical representation shown in Fig. 2.

The direction of the arrows indicates the direction of the influence between the various categories. By definition, an effect stems from a cause. The arrows connecting factors to effects are therefore unidirectional (Fig. 2A). Most of the associations between factors (hazard and 

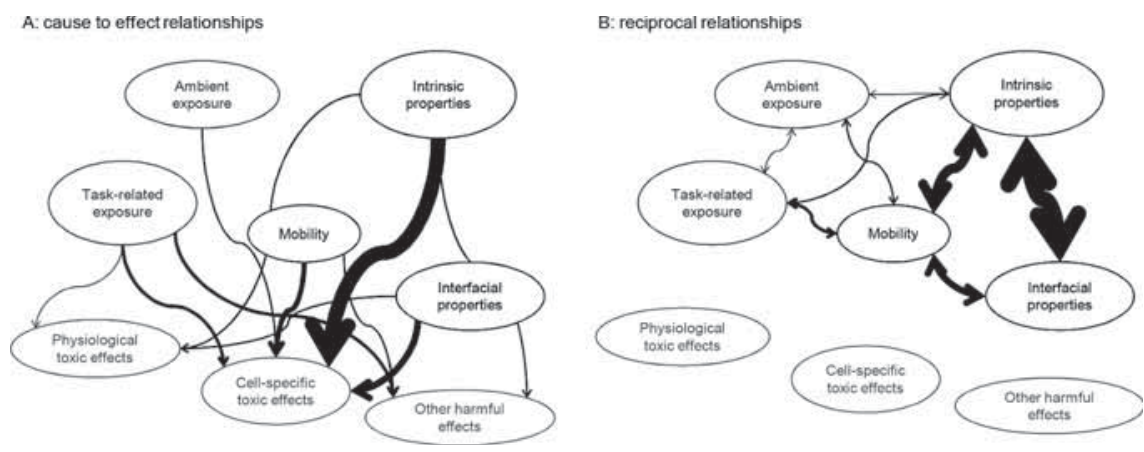

Figure 2: Hierarchised network of level-2 categories of elements of risk. (A) Cause to effect relationships and (B) reciprocal relationships.

exposure) are bi-directional, representing the interrelationship between the ENP hazard and transfer to the target (Fig. 2B). Each association between elements of risk (factors and effects) has been counted. As there is an association between particle size and uptake by cells, another between chemical composition and cell viability, then the score for these associations as a whole is 2 . The scores of each relationship among the eight level-2 categories are indicated in terms of arrow thickness. In other words, most associations join causes and effects, especially intrinsic properties or interfacial characteristics with effects on cells. This observation is no doubt due to the number of elements present in each level-2 category. However, for equivalent categories in relationship with a single category, ENP interfacial properties have been studied more than mobility properties. Some relationships could be established in both directions (e.g. influence of physical properties on exposure route and induction of changes in crystalline structure by UV) within the same categories, thus meaning the interrelationship between all ENP characteristics.

In addition, few relationships involving exposure factors have been established in the literature. This reflects the lack of exposure studies, as noted elsewhere [20, 43, 51], even though this type of study approaches field reality as experienced by workers and is therefore crucial. Other relationships also appearing less studied, indeed neglected, are those between mobility and general toxic effects. Direct connexions between ENP toxicokinetic effects and agglomeration effects should be examined. The representation obtained shows clearly the interdependence of the factors with each other as well as with effects. This observation confirms the complexity of the system and of the management of ENP-associated risks.

\section{DISCUSSION}

Since the focus of the present review is OHS, some parameters relevant to ENP products were not taken into consideration and do not appear in Table 1 . These are essentially parameters relating specifically to the environment and having little impact on OHS, at least in terms of direct contact between production site and workers. For example, transport, ecologic bioavailability, bioaccumulation and ecotoxicity (Table S2) could be taken into consideration in a study broadened to include the entire ENP lifecycle from production through commercial use and dispersion in the environment as a waste or spent product. In addition, since productive industrial businesses experiencing strong growth nanotechnologies stock relatively little product, it is reasonable to assume that ENP ageing represents a negligible risk for workers. On the other hand, biomimetic properties of ENPs, as reported by Gleich et al. [51], could be included in the list, but the science in this field is still embryonic. Another non-listed factor 
that would deserve consideration is the individual susceptibility but has never been referred previously in any risk assessment framework/review. One can logically think that disease or allergies may be a factor worth mentioning since ENP toxic effects might be enhanced for immune-compromised people.

In contrast with the work of Morgan [30] based on consultation of experts, the present review is based on data published between 2001 and 2011. One of the comments of this author referred to the lack of extensive scientific data. This observation should now be reexamined. The number of studies published on the subject of nanotechnologies and their effects on health has grown exponentially. However, another relatively recent study shows that in spite of this growth, several methodological and knowledge gaps remain [19]. In fact, both of these reports, based on consultation of experts, recommend improving and standardising ENP characterisation and toxicity tests. One of the aims of the present review was to produce a list of ENP-associated first-line risks, in other words focused specifically on the protection of workers. Another aim was to shed light on the value, the importance and the relevance of existing relationships between the various elements of risk, as reflected by the plethora of studies published during the period of interest. We thus note in particular, by way of simplified comparison, that certain categories of risk have been regarded in the scientific community as more important than others seemingly equally deserving of in-depth study. Morgan concluded her analysis with a similar remark [30]. Even without a precise enumeration and scientific evaluation of the studies relating to mobility parameters, this category of risk factor has apparently received relatively little attention.

This review, nevertheless, provides a graphical representation of the interdependence of all of the relevant factors and confirms the complexity of the ENP risk problem. This observation points to the structural character of the uncertainties involved. Structural uncertainty refers to the results obtained using a model of uncertain functional form rather than uncertainty regarding independent variables [97]. For example, while it is known that intrinsic and interfacial properties affect the cytotoxicity of a given ENP product, it is impossible to know if the same relationships are applicable to another type of ENP or if at least one parameter has been intentionally changed. This is because the variables are not independent, but interact.

In order to assess the accuracy and the rigour of our approach to managing risks associated with ENPs, we attempted to validate our results as well as the reliability of the method. It should be noted that the present review mostly provides observations and notes relating to ENPs. Internal validity refers to the validation of a conclusion between the dependent and the independent variables [98] and requires control of parasite variables and elimination of alternative explanations. At the present stage of development of ENP-associated OHS risk management, validation of the associations represented in Fig. 1 would require more detailed comparison of studies on the basis of specific criteria for a particular type of nanoparticle. In contrast, external validation [98] supports the generalisation of a conclusion to a system larger than the sample studied, for example, ENP products in general. The principal conclusion of the present review is that too much uncertainty remains for the validity of an association between any two level-2 or level-3 categories. This initial ranking of risks based on the literature is a first step towards ranking risks associated with synthetic nanoparticles. The fact that the large number of uncertainties limits the comparisons and, the relationships refers to some sort of external validation. Indeed, no relationship applying to any single ENP appears applicable to ENPs overall. This emphasises the need to use additional tools beyond review of the literature in order to rank these risks from an OHS perspective, such as a meta-analysis for example. 
As explained in Section 2 of this article, the literature review principles applied here were those described in previously published reviews. While conditions have of course evolved and our focus is different, the uncertainties noted in the present study are very similar to those noted in similar previous studies [19,30], which provides some support for the internal reliability [99] of our method. However, given the unique inherent complexity of ENPs, meta-analysis appears to be the best suited approach to evaluating external reliability.

Aside from the need for methods standardisation in order to improve the reliability of characterisation and toxicity evaluation as emphasised previously, other challenges remain as obstacles to effective risk management. The interdependence of all the system variables is certainly one of these. An interdisciplinary approach based on awareness of the impact of each parameter on other elements appears essential. Taking into consideration all of these interactions in the risk-ranking task will require the development of decision-aid tools based on original methods. Another major challenge will be integrating particle transfer [82]. In other words, as shown in Fig. 1, exposure factors are less studied than are intrinsic properties, even though it is the evaluation of these factors that will ultimately allow risk factor ranking that is both accurate and properly adapted to the studied environment.

Given the OHS focus of the present work, other risks associated with governance and communication should also be taken into account for a complete approach to risk management. The International Risk Governance Council [100] has examined the potential implications of nanotechnologies and the need to integrate these notions into a holistic approach to the management of risks associated with nanotechnologies.

\section{CONCLUSION AND PROSPECTS}

This review is a first step towards risk management in a specific context and is not intended as a ranking tool in itself. This work has nevertheless provided a detailed list of the risks associated with ENPs from an OHS perspective. Based on the fault tree method, a hierarchised risk network has been constructed revealing the overall system complexity due to the interdependence of the elements of risk. The graphical representation has also revealed deficiencies regarding the evaluation of certain risks, such as means of integrating exposure data.

Validation of the results obtained would benefit from consultation of experts to confirm observations made in the course of analysing the hierarchised risk network. In association with meta-analysis of associations between risk factors and effects and using a weight of evidence approach [101], this would enhance understanding of the relevance of risks for the purpose of protecting workers and OHS practitioners. Other steps, notably for integrating multi-sector data, are also necessary $[19,30]$.

Initial trials of a decision-aid tool have already been conducted [52]. Although multi-criteria methods are plausible choices for the management of ENP-associated risks, we question the capacity of this type of tool to tolerate the structural uncertainty inherent in such a system [31]. Several reports emphasise the necessity of developing an adaptive tool [37, 102]. This would involve the integration of toxicological data and field data in order to follow the constantly increasing flow of information about nanotechnologies and to ensure proper adaptation of monitoring and control measures intended to protect workers.

\section{ACKNOWLEDGEMENTS}

The authors wish to acknowledge $\mathrm{Ne}^{3} \mathrm{LS}$ network and the Institut de recherche Robert-Sauvé en santé et en sécurité du travail for funding this pilot study(IRSST report R-798). 


\section{LIST OF ABBREVIATIONS}

ADME: Absorption Distribution Metabolism and Excretion

ENP: Engineered Nanoparticles

ISO: International Standard Organization

OECD: Organization for Economic Co-operation and Development

OHS: Occupational Health and Safety

OSHA: Occupational Safety and Health Organization

REACH: Registration, Evaluation, Authorisation and Restriction of Chemical substances

SCENIHR: Scientific Committee on Emerging and Newly-Identified Health Risks

WHO: Word Health Organization

\section{REFERENCES}

[1] Nanotechproject. Project on Emerging Nanotechnologies. Inventories, available at http://www.nanotechproject.org/inventories/2011 (accessed 15 November 2011).

[2] The recession's ripple effect on nanotech: State of the market report. New-York: Lux Research Inc, 2009, June 9.

[3] Nanowerk. Databases/Company and Labs Directory, available at http://www. nanowerk.com/nanotechnology/research/nanotechnology_links.php2012 (accessed 6 January 2012).

[4] Johnson, D., Nanotech Employment Numbers Remain Inscrutable, available at http://spectrum.ieee.org/nanoclast/semiconductors/nanotechnology/nanotechemployment-numbers-remain-inscrutable-, 2010 (accessed 18 January 2012).

[5] Schulte, P., Geraci, C., Zumwalde, R., Hoover, M. \& Kuempel, E. Occupational risk management of engineered nanoparticles. Journal of Occupational and Environmental Hygiene, 5(4), pp. 239-249, 2008. doi: http://dx.doi.org/10.1080/15459620801907840

[6] OECD. Nanotechnology: an overview based on indicators and statistics. Organisation for Economic Co-operation and Development, 2009, Contract No.: 2009/7.

[7] Maynard, A. Don't define nanomaterials. Nature, 475, p. 31, 2011.

[8] Commission Recommendation of 18 october 2011 on the definition of nanomaterial. Report. Official Journal of the European Union: European Commission, 2011 October 18th. Report No.: Contract No.: 2011/696/EU.

[9] EPA. Control of Nanoscale Materials under the Toxic Substances Control Act, Washington, DC, available at http://www.epa.gov/oppt/nano/, 2011 (accessed 25 July 2012).

[10] WHO. Resolution II/4 on Emerging Policy Issues, available at http://www.who.int/ iomc/saicm/iccm2_resolution_II_4.pdf, 2009-2010 (accessed 25 July 2012).

[11] Registration, Evaluation, Authorisation and Restriction of Chemicals (REACH). Brussels, Belgium: European Commission, 2006 December 30th Report No.: Contract No.: 1907/2006.

[12] Morawska, L., Airborne engineered nanoparticles: are they a health problem? Air Quality and Climate Change, 44(3), p. 18, 2010.

[13] Morawska, L., Airborne particles and health. Air Quality and Climate Change, 44(2), pp. 13-15, 2010.

[14] Ostiguy, C., Roberge, B., Ménard, L. \& Endo, C-A., Best Practices Guide to Synthetic Nanoparticle Risk Management, Montreal, QC: Institut Robert Sauvé de Recherche en Santé et Sécurité au Travail, 2009 Contract No.: R-599.

[15] Ostiguy, C., Roberge, B., Woods, C. \& Soucy, B., Engineered Nanoparticles: Current Knowledge about Occupational Health and Safety Risks and Prevention Measures, 
2nd edn., Montreal, QC: Institut Robert Sauvé de Recherche en Santé et Sécurité au Travail, 2010 Contract No.: R-656.

[16] Oberdörster, G., Oberdörster, E. \& Oberdörster, J., Nanotoxicology: an emerging discipline evolving from studies of ultrafine particles. Environmental Health Perspectives, 113(7), pp. 823-839, 2005. doi: http://dx.doi.org/10.1289/ehp.7339

[17] Oberdorster, G., Maynard, A., Donaldson, K., Castranova, V., Fitzpatrick, J. \& Ausman, K. et al., Principles for characterizing the potential human health effects from exposure to nanomaterials: Elements of a screening strategy. Particle and Fibre Toxicology, 2, No pp given, 2005.

[18] Balbus, J.M., Maynard. A.D., Colvin, Vicki L., Castranova, V., Daston, G.P. \& Denison, R.A. et al., Meeting report: hazard assessment for nanoparticles--report from an interdisciplinary workshop. Environmental Health Perspectives, 115(11), pp. 1654-1659, 2007. doi: http://dx.doi.org/10.1289/ehp.10327

[19] Grieger, K.D., Hansen, S.F. \& Baun, A., The known unknowns of nanomaterials: describing and characterizing uncertainty within environmental, health and safety risks. Nanotoxicology, 3(3), pp. 222-233, 2009.

[20] Thomas, T., Bahadori, T., Savage, N. \& Thomas, K., Moving toward exposure and risk evaluation of nanomaterials: challenges and future directions. Wiley Interdisciplinary Reviews: Nanomedicine and Nanobiotechnology, 1(4), pp. 426-433, 2009.

[21] Aitken, R., Creely, K. \& Tran, C., Nanoparticles: An occupational hygiene review. Institute of Occupational Medicine, 2004 Contract No.: Research Report 274.

[22] Balbus, J.M., Florini. K. \& Denisond, R.A., Walsh SA. Getting it right the first time: developing nanotechnology while protecting workers, public health, and the environment. Annals of the New York Academy of Sciences, 1076, pp. 331-342, 2006. doi: http://dx.doi.org/10.1196/annals.1371.027

[23] ISO. Nanotechnologies - Health and safety practices in occupational settings relevant to nanotechnologies - ISO/TR 12885:2008. 2008.

[24] ISO. Nanotechnologies - Nanomaterial risk evaluation - ISO/TR 13121:2011. 2011.

[25] OECD. Report of the workshop on risk assessement of manufactured nanomaterials in a regulatory context. Organisation for Economic Co-operation and Development, 2010 Contract No.: ENV/JM/MONO(2010)10.

[26] OECD. Current developments/activities on the safety of manufactured nanomaterials. Organisation for Economic Co-operation and Development, 2011 Contract No.: ENV/JM/MONO(2011)12.

[27] Regulatory aspects of nanomaterials. Brussels, Belgium: European Commission, 2008 Contract No.: COM/2008/0366.

[28] Ostiguy, C., Roberge, B., Menard, L. \& Endo, C.A., A good practice guide for safe work with nanoparticles: the Quebec approach. Journal of Physics: Conference Series, 151, No pp given, 2009.

[29] Marchant, G.E., Sylvester, D.J. \& Abbott, K.W., Risk Management principles for nanotechnology. Nanoethics, 2, pp. 43-60, 2008.

[30] Morgan, K., Development of a preliminary framework for informing the risk analysis and risk management of nanoparticles. Risk Analysis 25(6), pp. 1621-1635, 2005. doi: http://dx.doi.org/10.1111/j.1539-6924.2005.00681.X

[31] Nadeau, S., Hallé, S. Viau, C, . \& Cloutier, Y., Call for the development of an adaptative tool to appreciate or evaluate risks to human health posed by synthetic nanoparticles. International Journal of Safety and Security Engineering, 2(1), pp. 40-53, 2012. doi: http://dx.doi.org/10.2495/SAFE-V2-N1-40-53 
[32] Kuzma, J., Paradise, J., Ramachandran, G., Kim, J-A., Kokotovich, A. \& Wolf, S.M., An integrated approach to oversight assessment for emerging technologies. Risk Analysis, 28(5), pp. 1197-1219, 2008. doi: http://dx.doi.org/10.1111/j.15396924.2008.01086.x

[33] Hansen, S.F., Maynard, A., Baun, A. \& Tickner, J.A., Late lessons from early warnings for nanotechnology. Nature Nanotechnology, 3(8), pp. 444-447, 2008. doi: http://dx. doi.org/10.1038/nnano.2008.198

[34] NAS. Risk Assessment in the Federal Government: Managing the Process, Washington, DC, 1983.

[35] Boote, D.N. \& Beile, P., Scholars before researchers: on the centrality of the dissertation literature review in research preparation. Educational Researcher, 34(6), pp. 3-15, 2005.

[36] Villemeur, A., Surete de fonctionnement des systemes industriels : fiabilite, facteurs humains, informatisation, Librairies Eyrolles and Électricité de France (EDF): Paris, France, 1988.

[37] Wardak, A., Gorman, M.E., Swami, N. \& Deshpande, S., Identification of risks in the life cycle of nanotechnology-based products. Journal of Industrial Ecology, 12(3), 435-448, 2008. doi: http://dx.doi.org/10.1111/j.1530-9290.2008.00029.x

[38] Warheit, D.B., Sayes, C.M., Reed, K.L. \& Swain, K.A., Health effects related to nanoparticle exposures: environmental, health and safety considerations for assessing hazards and risks. Pharmacology and Therapeutics, 120(1), pp. 35-42, 2008. doi: http://dx.doi.org/10.1016/j.pharmthera.2008.07.001

[39] Mortureux, Y., Arbres de défaillance, des causes et d'événement. Techniques de l'ingénieur. p. 24, 2002.

[40] Nadeau, S., Outil d'analyse multi-factorielle pour la prévention des lésions au dos, Universié de Montréal: Montréal, Canada, 2001.

[41] Grass, R.N., Limbach, L.K., Athanassiou, E.K. \& Stark, W.J., Exposure of aerosols and nanoparticle dispersions to in vitro cell cultures: a review on the dose relevance of size, mass, surface and concentration. Journal of Aerosol Science, 41(12), pp. 1123-1142, 2010. doi: http://dx.doi.org/10.1016/j.jaerosci.2010.10.001

[42] Oberdorster, G., Stone, V. \& Donaldson, K., Toxicology of nanoparticles: a historical perspective. Nanotoxicology, 1(1), pp. 2-25, 2007. doi: http://dx.doi.org/ $\underline{10.1080 / 17435390701314761}$

[43] SCENIHR. Risk Assessment of Products of Nanotechnologies. European Commission: Scientific Committee on Emerging and Newly Identified Health Risks, 2009 January 19.

[44] CEN. Workplace atmospheres: size fraction definitions for measurements of airborne particles in the workplace, Brussels, Belgium, 1993.

[45] Nanoscience and nanotechnologies: opportunities and uncertainties. London: Royal Society, 2004.

[46] Paik, S.Y., Zalk, D.M. \& Swuste, P., Application of a pilot control banding tool for risk level assessment and control of nanoparticle. The Annals of Occupational Hygiene, 52(6), pp. 419-428, 2008. doi: http://dx.doi.org/10.1093/annhyg/men041

[47] Berube, D., Cummings, C., Cacciatore, M., Scheufele, D. \& Kalin, J., Characteristics and classification of nanoparticles: expert Delphi survey. Nanotoxicology, 5(2), pp. 236-243, 2011. doi: http://dx.doi.org/10.3109/17435390.2010.521633

[48] EPA. Asbestos Mechanisms of Toxicity Workshop. Available at http://www.epa.gov/ oswer/asbestos_ws/summary.htm, 2003 (updated 26 December 2008; accessed 22 January 2012). 
[49] HCSP. Haut Conseil de la santé publique - Avis relatifà la sécurité des travailleurs lors de l'exposition aux nanotubes de carbone. France, available at http://www.hcsp.fr/docspdf/ avisrapports/hcspa20090107_ExpNanoCarbone.pdf, 2009 (accessed 1 September 2012).

[50] AFSSET. Évaluation des risques liés aux nanomatériaux pour la population générale et pour l'environnement. Agence Française de Sécurité Sanitaire de l'Environnement et du Travail: France, 2008 Contract No.: 2008/005.2008.

[51] von Gleich, A., Steinfeldt, M. \& Petschow, U., A suggested three-tiered approach to assessing the implications of nanotechnology and influencing its development. Journal of Cleaner Production, 16, pp. 899-909, 2008. doi: http://dx.doi.org/10.1016/j. jclepro.2007.04.017

[52] Linkov, I., Bates, M.E., Canis, L.J., Seager, T.P. \& Keisler, J.M., A decision-directed approach for prioritizing research into the impact of nanomaterials on the environment and human health. Nature Nanotechnology, 6(12), pp. 784-787. 2011. doi: http:// dx.doi.org/10.1038/nnano.2011.163

[53] Tsuji, J.S., Maynard, A.D., Howard, P.C., James, J.T., Lam, C-W. \& Warheit, D.B. et al., Research strategies for safety evaluation of nanomaterials, Part IV: risk assessment of nanoparticles. Toxicological Sciences, 89(1), pp. 42-50, 2006. doi: http://dx.doi.org/10.1093/toxsci/kfi339

[54] Borm, P.J.A., Robbins, D., Haubold, S., Kuhlbusch, T., Fissan, H. \& Donaldson. K. et al., The potential risks of nanomaterials: a review carried out for ECETOC. Particle and Fibre Toxicology, 3, No pp given, 2006.

[55] Lundqvist, M., Sethson, I. \& Jonsson, B-H., Protein adsorption onto silica nanoparticles: conformational changes depend on the particles' curvature and the protein stability. Langmuir, 20(24), pp. 10639-10647, 2004. doi: http://dx.doi. org/10.1021/la0484725

[56] Cedervall, T., Lynch, I., Foy, M., Berggard, T., Donnelly, S.C. \& Cagney, G. et al., Detailed identification of plasma proteins adsorbed on copolymer nanoparticles. Angewandte Chemie (International ed. in English), 46(30), pp. 5754-5756, 2007. doi: http://dx.doi.org/10.1002/anie.200700465

[57] Cedervall, T., Lynch, I., Lindman, S., Berggard, T., Thulin, E. \& Nilsson, H. et al., Understanding the nanoparticle-protein corona using methods to quantify exchange rates and affinities of proteins for nanoparticles. Proceedings of the National Academy of Sciences of the United States of America, 104(7), pp. 2050-2055, 2007. doi: http:// dx.doi.org/10.1073/pnas.0608582104

[58] Fubini, B., Ghiazza, M. \& Fenoglio, I., Physico-chemical features of engineered nanoparticles relevant to their toxicity. Nanotoxicology, 4(4), pp. 347-363, 2010. doi: http://dx.doi.org/10.3109/17435390.2010.509519

[59] Barillet, S., Simon-Deckers, A., Herlin-Boime, N., Mayne-L'Hermite, M., Reynaud, C. \& Cassio, D. et al., Toxicological consequences of TiO2, SiC nanoparticles and multi-walled carbon nanotubes exposure in several mammalian cell types: an in vitro study. Journal of Nanoparticle Research, 12(1), pp. 61-73, 2010. doi: http://dx.doi. org/10.1007/s11051-009-9694-y

[60] Auffan, M., Rose, J., Wiesner, M.R. \& Bottero, J-Y., Chemical stability of metallic nanoparticles: a parameter controlling their potential cellular toxicity in vitro. Environmental Pollution (Oxford, United Kingdom), 157(4), pp. 1127-1133, 2009.

[61] Derfus, A.M., Chan, W.C.W. \& Bhatia, S.N., Probing the cytotoxicity of semiconductor quantum dots. Nano Letters, 4(1), pp. 11-18, 2004. doi: http://dx.doi.org/10.1021/ $\underline{\text { n10347334 }}$ 
[62] Kittler, S., Greulich, C., Diendorf, J., Koeller, M. \& Epple, M., Toxicity of silver nanoparticles increases during storage because of slow dissolution under release of silver ions. Chemistry of Materials, 22(16), pp. 4548-4554, 2010. doi: http://dx.doi. org $/ 10.1021 / \mathrm{cm} 100023 \mathrm{p}$

[63] Lubick, N., Nanosilver toxicity: ions, nanoparticles-or both? Environmental Science and Technology, 42(23), p. 8617, 2008. doi: http://dx.doi.org/10.1021/es8026314

[64] Domingos, R.F., Baalousha, M.A., Ju-Nam, Y., Reid, M.M., Tufenkji, N. \& Lead, J.R. et al., Characterizing manufactured nanoparticles in the environment: multimethod determination of particle sizes. Environmental Science and Technology, 43(19), pp. 7277-7284, 2009. doi: http://dx.doi.org/10.1021/es900249m

[65] Verma, A. \& Stellacci, F., Effect of surface properties on nanoparticle-cell interactions. Small, 6(1), pp. 12-21, 2010. doi: http://dx.doi.org/10.1002/smll.200901158

[66] Guo, L., Liu, X., Sanchez, V., Vaslet, C., Kane, A.B. \& Hurt, R.H., A window of opportunity: designing carbon nanomaterials for environmental safety and health. Materials Science Forum, 544-545, pp. 16-23, 2007.

[67] Ostiguy, C., Lapointe, G., Ménard, L., Cloutier, Y., Trottier, M. \& Boutin, M. et al., Actual Knowledge about Occupational Health and Safety Risks and Prevention Measures, Institut Robert Sauvé de Recherche en Santé et Sécurité au Travail, 2006 Contract No.: R-470.

[68] Shatkin, J.A. \& Barry, B.E., Approaching risk assessment of nanoscale materials. NSTI Nanotech 2006, NSTI Nanotechnology Conference and Trade Show, Boston, MA, United States, May 7-11, 2006, 1, pp. 553-556, 2006.

[69] Schulte, P., Geraci, C., Zumwalde, R., Hoover, M. \& Kuempel, E., Occupational risk management of engineered nanoparticles. Journal of Occupational and Environmental Hygiene, 5, pp. 239-249, 2008. doi: http://dx.doi.org/10.1080/15459620801907840

[70] Schulte, P., Geraci, C., Zumwalde, R., Hoover, M., Castranova, V. \& Kuempel, E. et al., Sharpening the focus on occupational safety and health in nanotechnology. Scandinavian Journal of Work, Environment \& Health, 34(6), pp. 471-478, 2008. doi: http://dx.doi.org/10.5271/sjweh.1292

[71] O'Shaughnessy, P.T., Occupational health hazards of nanoparticles. In Nanoscience and Nanotechnology: Environmental and Health Impacts, ed. V.H. Grassian, John Wiley \& Sons, Inc.: Hoboken, NJ, USA, pp. 427-460, 2008. doi: http://dx.doi. org/10.1002/9780470396612.ch17

[72] Murashov, V., Engel, S., Savolainen, K., Fullam, B., Lee, M. \& Kearns, P., Occupational safety and health in nanotechnology and Organisation for Economic Cooperation and Development. Journal of Nanoparticle Research, 11, pp. 1587-1591, 2009. doi: http://dx.doi.org/10.1007/s11051-009-9637-7

[73] Rozhkova, E.A., Ulasov, I., Lai, B., Dimitrijevic, N.M., Lesniak, M. \& Rajh, T., A high-performance nanobio photocatalyst for targeted brain cancer therapy. Nano Letters, 9(9), pp. 3337-3342, 2009. doi: http://dx.doi.org/10.1021/n1901610f

[74] Johnston, H.J., Hutchison, G.R., Christensen, F.M., Peters, S., Hankin, S. \& Stone, V., Identification of the mechanisms that drive the toxicity of $\mathrm{TiO} 2$ particulates: the contribution of physicochemical characteristics. Particle and Fibre Toxicology, 6, 2009.

[75] Kouam, J., Songmene, V., Djebara, A. \& Khettabi, R., Effect of friction testing of metals on particle emission. Journal of Materials Engineering and Performance, 21(6), pp. 965-972, 2012.

[76] Kuhlbusch, T.A., Asbach, C., Fissan, H., Gohler, D. \& Stintz, M., Nanoparticle exposure at nanotechnology workplaces: a review. Particle and Fibre Toxicology, 8, p. 22, 2011. doi: http://dx.doi.org/10.1186/1743-8977-8-22 
[77] Kandlikar, M., Ramachandran, G., Maynard Andrew, D. \& Murdock, B., Health risk assessment for nanoparticles: a case for using expert judgment. Journal of Nanoparticle Research, 9, pp. 137-156, 2007. doi: http://dx.doi.org/10.1007/s11051006-9154-X

[78] Oberdorster, G., Sharp, Z., Elder, A.P., Gelein, R., Kreyling, W.G. \& Cox, C., Translocation of inhaled ultrafine particles to the brain. Inhalation Toxicology, 16, pp. 437-445, 2004. doi: http://dx.doi.org/10.1080/08958370490439597

[79] Aschberger, K., Micheletti, C., Sokull-Kluettgen, B. \& Christensen, F.M., Analysis of currently available data for characterising the risk of engineered nanomaterials to the environment and human health - lessons learned from four case studies. Environment International, 37(6), pp. 1143-1156, 2011. doi: http://dx.doi.org/10.1016/j.envint. 2011.02.005

[80] SCENIHR. The appropriateness of the risk assessment methodology in accordance with the technical guidance documents for new and existing substances for assessing the risks of nanomaterials. European Commission: Scientific Committee on Emerging and Newly-Identified Health Risks, 2007 June 21-22.

[81] Becker, H., Herzberg, F., Schulte, A. \& Kolossa-Gehring, M., The carcinogenic potential of nanomaterials, their release from products and options for regulating them. International Journal of Hygiene and Environmental Health, 214(3), pp. 231-238, 2011. doi: http://dx.doi.org/10.1016/j.ijheh.2010.11.004

[82] Binet, S., Drais, E., Chazelet, S., Fontaine, J-R., Radauceanu, A. \& Reynier, M. et al., Risques liés aux nanoparticules et nanomatériaux: Compte-rendu de la conférence Nano2011 et perspectives.: INRS: France, 2011 Contract No.: HST 224-16.

[83] Nemmar, A., Vanbilloen, H., Hoylaerts, M.F., Hoet, P.H., Verbruggen, A. \& Nemery, B., Passage of intratrachelly instilled ultrafine particles from the lung into the systemic circulation in hamster. American Journal of Respiratory and Critical Care Medicine, 164, pp. 1665-1668, 2001. doi: http://dx.doi.org/10.1164/ajrccm.164.9.2101036

[84] Auffan, M., Rose, J., Bottero, J-Y., Lowry, G.V., Jolivet, J-P. \& Wiesner, M.R., Towards a definition of inorganic nanoparticles from an environmental, health and safety perspective. Nature Nanotechnology, 4(10), pp. 634-641, 2009. doi: http:// dx.doi.org/10.1038/nnano.2009.242

[85] Borm, P., Klaessig, F.C., Landry, T.D., Moudgil, B., Pauluhn, J. \& Thomas, K. et al., Research strategies for safety evaluation of nanomaterials, Part V: role of dissolution in biological fate and effects of nanoscale particles. Toxicological Sciences, $\mathbf{9 0 ( 1 ) , ~ p p . ~}$ 23-32, 2006. doi: http://dx.doi.org/10.1093/toxsci/kfj084

[86] Vertegel, A.A., Siegel, R.W. \& Dordick, J.S., Silica nanoparticle size influences the structure and enzymatic activity of adsorbed lysozyme. Langmuir, 20(16), pp. 6800-6807, 2004. doi: http://dx.doi.org/10.1021/la0497200

[87] Lord, M.S., Cousins, B.G., Doherty, P.J., Whitelock, J.M., Simmons, A. \& Williams, R.L. et al., The effect of silica nanoparticulate coatings on serum protein adsorption and cellular response. Biomaterials, 27, pp. 4856-4862, 2006. doi: http://dx.doi.org/10.1016/j. biomaterials.2006.05.037

[88] Chithrani, B.D. \& Chan, W.C.W., Elucidating the mechanism of cellular uptake and removal of protein-coated gold nanoparticles of different sizes and shapes. Nano Letters, 7(6), pp. 1542-1550, 2007. doi: http://dx.doi.org/10.1021/n1070363y

[89] Clift, M.J.D., Rothen-Rutishauser, B., Brown, D.M., Duffin, R., Donaldson, K. \& Proudfoot, L. et al., The impact of different nanoparticle surface chemistry and size on uptake and toxicity in a murine macrophage cell line. Toxicology and 
Applied Pharmacology, 232(3), pp. 418-427, 2008. doi: http://dx.doi.org/10.1016/j. taap.2008.06.009

[90] Braydich-Stolle, L.K., Schaeublin, N.M., Murdock, R.C., Jiang, J., Biswas, P. \& Schlager, J.J. et al., Crystal structure mediates mode of cell death in $\mathrm{TiO} 2$ nanotoxicity. Journal of Nanoparticle Research, 11(6), pp. 1361-1374, 2009. doi: http://dx.doi. org/10.1007/s11051-008-9523-8

[91] Gratton, S.E.A., Ropp, P.A., Pohlhaus, P.D., Luft, J.C., Madden, V.J. \& Napier, M.E. et al., The effect of particle design on cellular internalization pathways. Proceedings of the National Academy of Sciences of the United States of America, 105(33), pp. 11613-11618, 2008. doi: http://dx.doi.org/10.1073/pnas.0801763105

[92] Hoshino, A., Fujioka, K., Oku, T., Suga, M., Sasaki, Y.F. \& Ohta, T. et al., Physicochemical properties and cellular toxicity of nanocrystal quantum dots depend on their surface modification. Nano Letters, 4(11), pp. 2163-2169, 2004. doi: http:// dx.doi.org/10.1021/n1048715d

[93] Papageorgiou, I., Brown, C., Schins, R., Singh, S., Newson, R. \& Davis, S. et al., The effect of nano- and micron-sized particles of cobalt-chromium alloy on human fibroblasts in vitro. Biomaterials, 28(19), pp. 2946-2958, 2007. doi: http://dx.doi. org/10.1016/j.biomaterials.2007.02.034

[94] Simko, M. \& Mattsson, M-O., Risks from accidental exposures to engineered nanoparticles and neurological health effects: a critical review. Particle and Fibre Toxicology, 7, p. 42, 2010. doi: http://dx.doi.org/10.1186/1743-8977-7-42

[95] Gonzalez, L., Lison, D. \& Kirsch-Volders, M., Genotoxicity of engineered nanomaterials: a critical review. Nanotoxicology, 2(4), pp. 252-273, 2008. doi: http:// dx.doi.org/10.1080/17435390802464986

[96] Kreyling, W.G., Semmler, M., Erbe, F., Mayer, P., Takenaka, S. \& Schulz, H. et al., Translocation of ultrafine insoluble iridium particles from lung epithelium to extrapulmonary organs is size dependent but very low. Journal of Toxicology and Environmental Health, Part A, 65(20), pp. 1513-1530, 2002. doi: http://dx.doi. org/10.1080/00984100290071649

[97] Strong, M., Oakley, J.E. \& Chilcott, J., Managing structural uncertainty in health economic decision models: a discrepancy approach. Applied Statistics, 61, pp. 25-45, 2012. doi: http://dx.doi.org/10.1111/j.1467-9876.2011.01014.x

[98] Leedy, P.D. \& Ormrod, J.L., Practical Research: Planning and Design, 9th edn., Merril: Upper Saddle River, New Jersey, 2010.

[99] Gagnon, Y-C., L'étude de cas comme méthode de recherche, Presses de l'Université du Québec: Québec, 2005.

[100] Nanotechnology risk governance. International Risk Governance Council, 2007.

[101] Zuin, S., Micheletti, C., Critto, A., Pojana, G., Johnston, H. \& Stone, V. et al., Weight of Evidence approach for the relative hazard ranking of nanomaterials. Nanotoxicology, 5(3), pp. 445-458, 2011. doi: http://dx.doi.org/10.3109/17435390.2010.512986

[102] Murashov ,V. \& Howard, J., Essential features for proactive risk management. Nature Nanotechnology, 4(8), pp. 467-470, 2009. doi: http://dx.doi.org/10.1038/ nnano.2009.205

[103] Hansen, S.F., Larsen, B.H., Olsen, S.I. \& Baun, A., Categorization framework to aid hazard identification of nanomaterials. Nanotoxicology, 1(3), pp. 243-50, 2007. doi: http://dx.doi.org/10.1080/17435390701727509

[104] Singh, N., Manshian, B., Jenkins, G.J.S., Griffiths, S.M., Williams, P.M. \& Maffeis, T.G.G. et al., NanoGenotoxicology: the DNA damaging potential of engineered 
nanomaterials. Biomaterials, 30(23-24), pp. 3891-3914, 2009. doi: http://dx.doi. org/10.1016/j.biomaterials.2009.04.009

[105] Woskie, S., Workplace practices for engineered nanomaterial manufacturers. Wiley Interdisciplinary Reviews: Nanomedicine and Nanobiotechnology, 2(6), pp. 685-692, 2010.

[106] Linkov, I., Satterstrom, F.K., Steevens, J., Ferguson, E. \& Pleus, R.C., Multi-criteria decision analysis and environmental risk assessment for nanomaterials. Journal of Nanoparticle Research, 9, pp. 543-554, 2007. doi: http://dx.doi.org/10.1007/s11051$\underline{007-9211-0}$

\section{ELECTRONIC SUPPLEMENTARY INFORMATION}

Table S1: Matrix form of links between third-level classification risk elements identified over a large selection of literature between 2001 and 2011.

Table S2: List of other ENP-associated risk elements identified over a large selection of literature between 2001 and 2011 but not included in our classification. 